\title{
Mixtures of Experts Estimate A Posteriori Probabilities
}

\author{
Perry Moerland \\ IDIAP, CP 592, 1920 Martigny, Switzerland
}

\begin{abstract}
The mixtures of experts (ME) model offers a modular structure suitable for a divide-and-conquer approach to pattern recognition. It has a probabilistic interpretation in terms of a mixture model, which forms the basis for the error function associated with MEs. In this paper, it is shown that for classification problems the minimization of this $\mathrm{ME}$ error function leads to ME outputs estimating the a posteriori probabilities of class membership of the input vector.
\end{abstract}

\section{Introduction}

It is well-known that for artificial neural networks trained by minimizing sum-ofsquares or cross-entropy error functions for a classification problem, the network outputs approximate the a posteriori probabilities of class membership [2]. This property is a very useful one, especially when the network outputs are to be used in a further decision-making stage (e.g. rejection thresholds) or integrated in other statistical pattern recognition methods (as in hybrid NN-HMMs).

Recently, a modular architecture of neural networks known as a mixture of experts (ME) has attracted quite some attention [6][7]. MEs are mixture models which attempt to solve problems using a divide-and-conquer strategy; that is, they learn to decompose complex problems in simpler subproblems. In particular, the gating network of a ME learns to partition the input space (in a soft way, so overlaps are possible) and attributes expert networks to these different regions. The divide-and-conquer approach has shown particularly useful in attributing experts to different regimes in piece-wise stationary time series [9] and modeling discontinuities in the input-output mapping.

Mixtures of experts have also been successfully applied to classification problems [4][8], though a proof that minimization of the ME error function (based on the formulation as a mixture model) leads to ME outputs estimating the a posteriori probabilities of class membership, is still lacking. The purpose of this paper is to show that at the global minimum of this ME error function, the ME outputs do indeed estimate a posteriori probabilities.

\section{Mixtures of Experts}

In this section the basic definitions of the mixture of experts model are given which will be used in the rest of the paper. 


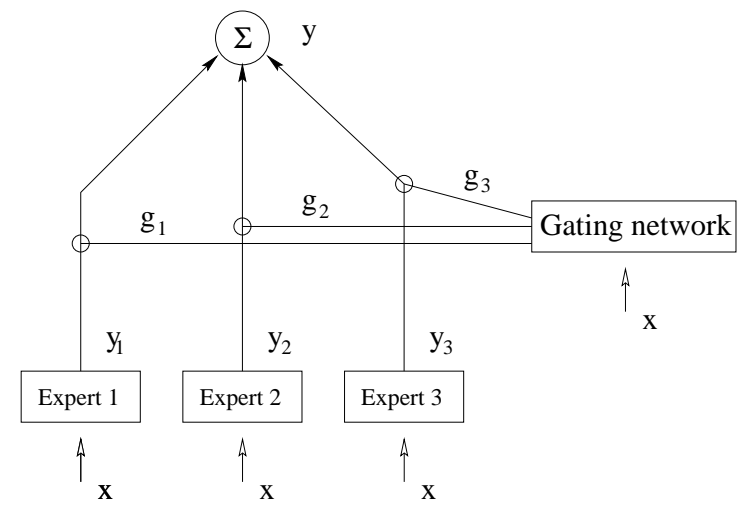

Figure1. Architecture of a mixture of experts network.

Figure 1 shows the architecture of a ME network, consisting of three expert networks and one gating network both having access to the input vector $\mathbf{x}$; the gating network has one output $g_{i}$ per expert. The standard choices for gating and expert networks are generalized linear models [7] and multilayer perceptrons [9]. The output vector of a ME is the weighted (by the gating network outputs) mean of the expert outputs:

$$
\mathbf{y}(\mathbf{x})=\sum_{j=1}^{m} g_{j}(\mathbf{x}) \mathbf{y}_{j}(\mathbf{x})
$$

The gating network outputs $g_{j}(\mathbf{x})$ can be regarded as the probability that input $\mathbf{x}$ is attributed to expert $j$. In order to ensure this probabilistic interpretation, the activation function for the outputs of the gating network is chosen to be the soft-max function [3]:

$$
g_{j}=\frac{\exp \left(z_{j}\right)}{\sum_{i=1}^{m} \exp \left(z_{i}\right)},
$$

where the $z_{i}$ are the gating network outputs before thresholding. This soft-max function makes that the gating network outputs sum to unity and are nonnegative; thus implementing the (soft) competition between the experts.

A probabilistic interpretation of a ME can be given in the context of mixture models for conditional probability distributions (see section 6.4 in [1]):

$$
p(\mathbf{t} \mid \mathbf{x})=\sum_{j=1}^{m} g_{j}(\mathbf{x}) \phi_{j}(\mathbf{t} \mid \mathbf{x}),
$$

where the $\phi_{j}$ represent the conditional densities of target vector $\mathbf{t}$ for expert $j$. The use of a soft-max function in the gating network and the fact that the $\phi_{j}$ are densities guarantee that the distribution is normalized: $\int p(\mathbf{t} \mid \mathbf{x}) d \mathbf{t}=1$.

As outlined in the next section this distribution forms the basis for the ME error function which can be optimized using gradient descent or the ExpectationMaximization (EM) algorithm [7]. 


\section{Estimating Posterior Probabilities}

A standard way to motivate error functions is from the principle of maximum likelihood of the (independently distributed) training data $\left\{\mathbf{x}^{n}, \mathbf{t}^{n}\right\}$ (see section 6.1 in [1]):

$$
\mathcal{L}=\prod_{n} p\left(\mathbf{x}^{n}, \mathbf{t}^{n}\right)=\prod_{n} p\left(\mathbf{t}^{n} \mid \mathbf{x}^{n}\right) p\left(\mathbf{x}^{n}\right)
$$

A cost function is then obtained by taking the negative logarithm of the likelihood (and dropping the term $p\left(\mathbf{x}^{n}\right)$ which does not depend on the network parameters):

$$
E=-\sum_{n} \ln p\left(\mathbf{t}^{n} \mid \mathbf{x}^{n}\right)
$$

The most suitable choice for the conditional probability density depends on the problem. For regression problems a Gaussian noise model is appropriate (leading to the sum-of-squares error function); for classification problems with a 1-of- $c$ coding scheme and outputs $y_{c}$ for each class, a multinomial density is most suitable:

$$
p\left(\mathbf{t}^{n} \mid \mathbf{x}^{n}\right)=\prod_{c=1}^{C}\left(y_{c}^{n}\right)^{t_{c}^{n}} .
$$

This offers us the framework to obtain a cost function for the mixtures of experts model. In its most general form the ME error function to be minimized is (substituting (3) in (4)):

$$
E=-\sum_{n} \ln \sum_{j=1}^{m} g_{j}\left(\mathbf{x}^{n}\right) \phi_{j}\left(\mathbf{t}^{n} \mid \mathbf{x}^{n}\right)
$$

the exact formulation of which depends on the choice for the conditional densities $\phi_{j}\left(\mathbf{t}^{n} \mid \mathbf{x}^{n}\right)$. Since, our main interest is in MEs for classification problems, the $\phi_{j}$ are assumed to be multinomial densities (5) in the rest of this paper. As in the gating network of a ME, a suitable choice for the activation function for the expert output units is then the soft-max function (2):

$$
y_{j c}=\frac{\exp \left(a_{j c}\right)}{\sum_{k} \exp \left(a_{j k}\right)},
$$

where the $a_{j c}$ are the expert network outputs before thresholding.

In the limit of an infinite data set (to avoid bias and variance) the finite sum over patterns can be replaced with an integral:

$$
E=-\iint \ln \left(\sum_{j=1}^{m} g_{j}(\mathbf{x}) \phi_{j}(\mathbf{t} \mid \mathbf{x})\right) p(\mathbf{t}, \mathbf{x}) d \mathbf{t} d \mathbf{x},
$$

factoring the joint distribution:

$$
E=-\iint \ln \left(\sum_{j=1}^{m} g_{j}(\mathbf{x}) \phi_{j}(\mathbf{t} \mid \mathbf{x})\right) p(\mathbf{t} \mid \mathbf{x}) p(\mathbf{x}) d \mathbf{t} d \mathbf{x}
$$


The interpretation of the ME outputs when this error function is minimized, can be obtained by setting to zero the functional derivatives [5] of $E$ with respect to the gating network outputs $z_{j}(\mathbf{x})$ and the network outputs of expert $j, a_{j c}(\mathbf{x})$. The solution of these equations will then result in expressions for $g_{j}(\mathbf{x})$ and $\mathbf{y}_{j}(\mathbf{x})$ at the minimum of $E$ (along the lines of section 6.1 .3 of [1] for the sum-of-squares error function).

Defining:

$$
E^{\prime}=\ln \sum_{j=1}^{m} g_{j}(\mathbf{x}) \phi_{j}(\mathbf{t} \mid \mathbf{x})
$$

we are then interested in the following two functional derivatives set to zero. For the gating network:

$$
\frac{\delta E}{\delta z_{j}}=-\int\left(\frac{\partial E^{\prime}}{\partial z_{j}}\right) p(\mathbf{t} \mid \mathbf{x}) p(\mathbf{x}) d \mathbf{t}=0
$$

and for the expert network (using the chain rule):

$$
\frac{\delta E}{\delta a_{j c}}=-\int\left(\frac{\partial E^{\prime}}{\partial a_{j c}}\right) p(\mathbf{t} \mid \mathbf{x}) p(\mathbf{x}) d \mathbf{t}=-\int \sum_{k} \frac{\partial E^{\prime}}{\partial y_{j k}} \frac{\partial y_{j k}}{\partial a_{j c}} p(\mathbf{t} \mid \mathbf{x}) p(\mathbf{x}) d \mathbf{t}=0
$$

In section 6.4 of [1], the partial derivatives for the gating network occurring in (8) have been calculated in the context of a gradient descent algorithm for the mixture model (3). Bishop's outcomes are restated here:

$$
\frac{\partial E^{\prime}}{\partial z_{j}}=\sum_{k} \frac{\partial E^{\prime}}{\partial g_{k}} \frac{\partial g_{k}}{\partial z_{j}}=\sum_{k}-\frac{\pi_{k}}{g_{k}}\left(\delta_{j k} g_{k}-g_{j} g_{k}\right)=g_{j}-\pi_{j}
$$

where the posterior probability $\pi_{j}$ is defined as:

$$
\pi_{j}(\mathbf{x}, \mathbf{t})=\frac{g_{j} \phi_{j}}{\sum_{i} g_{i} \phi_{i}}
$$

and $\delta_{j k}$ is the Kronecker delta. The functional derivative set zero with respect to the gating network outputs is (substituting (10) in (8)):

$$
\frac{\delta E}{\delta z_{j}}=-\int\left(g_{j}-\pi_{j}\right) p(\mathbf{t} \mid \mathbf{x}) p(\mathbf{x}) d \mathbf{t}=0 .
$$

The solution of the expert network equation (9) will be treated in some more detail. Recall that for classification problems, the expert outputs are obtained with a soft-max function (6). Therefore, the second partial derivative, $\partial y_{j k} / \partial a_{j c}$, in (9) is similar to its counterpart in the gating network equation $\partial g_{k} / \partial z_{j}$ (see the second term in $(10))$ :

$$
\frac{\partial y_{j k}}{\partial a_{j c}}=\delta_{c k} y_{j k}-y_{j c} y_{j k}
$$


Using the definition of $E^{\prime}(7)$ and of the multinomial density $\phi_{j}(5)$ gives for the first partial derivative in (9):

$$
\frac{\partial E^{\prime}}{\partial y_{j k}}=\frac{\partial\left(\ln \sum_{j=1}^{m} g_{j} \phi_{j}\right)}{\partial y_{j k}}=\frac{\partial\left(\ln \sum_{j=1}^{m} g_{j} \prod_{c=1}^{C}\left(y_{j c}\right)^{t_{c}}\right)}{\partial y_{j k}},
$$

that is, taking the partial derivative and using (11):

$$
\frac{\partial E^{\prime}}{\partial y_{j k}}=\frac{g_{j}\left(y_{j k}\right)^{\left(t_{k}-1\right)} t_{k}}{\sum_{i=1}^{m} g_{i} \phi_{i}} \prod_{c=1, c \neq k}^{C}\left(y_{j c}\right)^{t_{c}}=\frac{g_{j} \phi_{j}}{\sum_{i=1}^{m} g_{i} \phi_{i}} \frac{t_{k}}{y_{j k}}=\pi_{j} \frac{t_{k}}{y_{j k}} .
$$

Preparing for the solution of (9) one needs (using (13) and (14)):

$$
\sum_{k} \frac{\partial E^{\prime}}{\partial y_{j k}} \frac{\partial y_{j k}}{\partial a_{j c}}=\sum_{k} \pi_{j}(\mathbf{x}, \mathbf{t}) \frac{t_{k}}{y_{j k}}\left(\delta_{c k} y_{j k}-y_{j c} y_{j k}\right)=\pi_{j}(\mathbf{x}, \mathbf{t}) t_{c}-\pi_{j}(\mathbf{x}, \mathbf{t}) y_{j c}
$$

where in the last step it has been used that for 1-of- $c$ classification problems, $\sum_{k} t_{k}=1$. The functional derivative set to zero with respect to the expert network outputs is (substituting (15) in (9)):

$$
\frac{\delta E}{\delta a_{j e}}=-\int\left(\pi_{j}(\mathbf{x}, \mathbf{t}) t_{c}-\pi_{j}(\mathbf{x}, \mathbf{t}) y_{j c}\right) p(\mathbf{t} \mid \mathbf{x}) p(\mathbf{x}) d \mathbf{t}=0 .
$$

What is left is to determine the $g_{j}(\mathbf{x})$ and $y_{j}(\mathbf{x})$ that solve (12) and (16) (and therefore minimize the ME error function). For the gating network outputs (12):

$$
\frac{\delta E}{\delta z_{j}}=-g_{j} p(\mathbf{x}) \int p(\mathbf{t} \mid \mathbf{x}) d \mathbf{t}+p(\mathbf{x}) \int \pi_{j}(\mathbf{x}, \mathbf{t}) p(\mathbf{t} \mid \mathbf{x}) d \mathbf{t}=0 .
$$

using that the conditional probability $p(\mathbf{t} \mid \mathbf{x})$ is normalized:

$$
\frac{\delta E}{\delta z_{j}}=-g_{j} p(\mathbf{x})+p(\mathbf{x}) \int \pi_{j}(\mathbf{x}, \mathbf{t}) p(\mathbf{t} \mid \mathbf{x}) d \mathbf{t}=0 .
$$

Therefore, at the minimum of the ME error function the gating network outputs satisfy:

$$
g_{j}=\int \pi_{j}(\mathbf{x}, \mathbf{t}) p(\mathbf{t} \mid \mathbf{x}) d \mathbf{t} .
$$

For the expert network outputs (16):

$$
\frac{\delta E}{\delta a_{j c}}=-p(\mathbf{x}) \int \pi_{j}(\mathbf{x}, \mathbf{t}) t_{c} p(\mathbf{t} \mid \mathbf{x}) d \mathbf{t}+y_{j c} p(\mathbf{x}) \int \pi_{j}(\mathbf{x}, \mathbf{t}) p(\mathbf{t} \mid \mathbf{x}) d \mathbf{t}=0 .
$$

Therefore, at the minimum of the ME error function the expert network outputs satisfy:

$$
y_{j c}=\frac{\int \pi_{j}(\mathbf{x}, \mathbf{t}) t_{c} p(\mathbf{t} \mid \mathbf{x}) d \mathbf{t}}{\int \pi_{j}(\mathbf{x}, \mathbf{t}) p(\mathbf{t} \mid \mathbf{x}) d \mathbf{t}}
$$


Finally, using (17) and (18), the output vector of a mixture of experts that minimizes the ME error function is (1):

$$
y_{c}(\mathbf{x})=\sum_{j} g_{j}(\mathbf{x}) y_{j c}(\mathbf{x})=\sum_{j} \int \pi_{j}(\mathbf{x}, \mathbf{t}) t_{c} p(\mathbf{t} \mid \mathbf{x}) d \mathbf{t},
$$

exchanging integration and summation:

$$
\int \sum_{j} \pi_{j}(\mathbf{x}, \mathbf{t}) t_{c} p(\mathbf{t} \mid \mathbf{x}) d \mathbf{t}=\int t_{c} p(\mathbf{t} \mid \mathbf{x}) d \mathbf{t}:=\left\langle t_{c} \mid \mathbf{x}\right\rangle,
$$

where we have used that the posterior probabilities $\pi_{j}(\mathbf{x}, \mathbf{t})(11)$ sum to unity. The interpretation of (19) is that the output $y_{c}(\mathbf{x})$ of a ME at the minimum of the ME error function is equal to the conditional average of the target data. This is exactly the same as for the outputs of a network trained by minimizing the sum-of-squares or cross-entropy error functions [1]. It is a well-known result that for a classification problem with 1-of- $c$ coding the conditional average of the target data is (see, for example, section 6.6 in [1]) :

$$
y_{c}(\mathbf{x})=P\left(\mathcal{C}_{c} \mid \mathbf{x}\right),
$$

so that the outputs of a ME do indeed estimate the a posteriori probability that $\mathbf{x}$ belongs to class $\mathcal{C}_{c}$.

\section{Discussion}

In section 3 , it was assumed that the conditional density $\phi_{j}\left(\mathbf{t}^{n} \mid \mathbf{x}^{n}\right)$ of expert $j$ is multinomial. However, this is not a necessary condition for ME to estimate a posteriori probabilities. It can be shown that also a Gaussian noise model:

$$
\phi_{j}\left(\mathbf{t}^{n} \mid \mathbf{x}^{n}\right)=\frac{1}{(2 \pi)^{(c / 2)}} \exp \left(-\frac{\left\|\mathbf{t}-\mathbf{y}_{j}(\mathbf{x})\right\|^{2}}{2}\right)
$$

leads to this result.

\section{Acknowledgments}

The author gratefully acknowledges the Swiss National Science Foundation (FN:21-45621.95) for their support of this research.

\section{References}

1. Christopher M. Bishop. Neural Networks for Pattern Recognition. Oxford University Press, Oxford, 1995. 
2. H. Bourlard and N. Morgan. Links between Markov models and multi-layer perceptrons. In D. S. Touretzky, editor, Advances in Neural Information Processing, volume 1, pages 502-510, San Mateo CA, 1989. Morgan Kaufmann.

3. J. S. Bridle. Probabilistic interpretation of feedforward classification network outputs with relationships to statistical pattern recognition. In F. Fogelman Soulié and J. Hérault, editors, Neurocomputing: Algorithms, Architectures, and Applications, pages 227-236. Springer Verlag, New York, 1990.

4. Jürgen Fritsch, Michael Finke, and Alex Waibel. Context-dependent hybrid HME/HMM speech recognition using polyphone clustering decision trees. In Proceedings of ICASSP-97, 1997.

5. Mariano Giaquinta and Stefan Hildebrandt. Calculus of Variations. Springer Verlag, Berlin, 1996.

6. Robert A. Jacobs, Michael I. Jordan, Steven J. Nowlan, and Geoffrey E. Hinton. Adaptive mixtures of local experts. Neural Computation, 3(1):79-87, 1991.

7. Michael I. Jordan and Robert A. Jacobs. Hierarchical mixtures of experts and the EM algorithm. Neural Computation, 6(2):181-214, 1994.

8. S. R. Waterhouse and A. J. Robinson. Classification using hierarchical mixtures of experts. In Proceedings 1994 IEEE Workshop on Neural Networks for Signal Processing, pages 177-186, Long Beach CA, 1994. IEEE Press.

9. Andreas S. Weigend, Morgan Mangeas, and Ashok N. Srivastava. Nonlinear gated experts for time series: Discovering regimes and avoiding overfitting. International Journal of Neural Systems, 6:373-399, 1995. 